\title{
Linguistic Barriers as a Hindrance to Information Flow: HIV and AIDS Cases in Botswana
}

\author{
Tacheba Colson B. Tacheba \\ Botho University, Boseja, Maun
}

\begin{abstract}
Several attempts have been made to combat the HIV and AIDS pandemic through Information Education Communication in Botswana. However, most of the information is predominantly in English in which the majority of the people are nonproficient. A primary obstacle therefore has been lack of comprehensive information education communication about HIV and AIDS pandemic in other local languages. The information education communication by Government, Non-governmental Organizations (NGOs) and community based organizations in Botswana using the Language of the people is therefore imperative as an empowering tool and vehicle for a viable social and behavioral change.
\end{abstract}

\section{Introduction}

Botswana has an estimated population of 2 million, $75 \%$ of which are in sexually active life. Out of the entire population, only $15 \%$ have gone for HIV testing and counseling, therefore the majority are not aware of their status [38]. According to National AIDS Coordinating Agency (NACA), HIV and AIDS prevalence in Botswana is at $14.1 \%$ representing over 600,000 people infected by HIV and AIDS [34]. Half of the total number are young people aged between $15-24$ years, $75 \%$ of these are girls. NACA also states that $16.9 \%$ of women attending antenatal clinics were HIV positive and most of them were below 30 years. HIV and AIDS prevalence is higher in urban areas, $21.1 \%$ and lower in rural Botswana, $12.1 \%$. This shows that HIV and AIDS prevalence is almost twice as high in urban areas as it is in rural areas. However studies suggest that prevalence is declining in many urban areas and rising in many rural ones [38].

Levels of infection in Botswana show that over half a million Batswana and one in every seven adults are infected with HIV and AIDS [39]. Of the national adult (15-49 age bracket) HIV prevalence, 56\% are women. Annual deaths due to HIV and AIDS are estimated at 87,000. The AIDS epidemic in Botswana has caused over half a million deaths so far and continues to be responsible for the deaths of around ten people every hour [38]. AIDS is a major factor in the country's low life expectancy of just 38.5 years. [31]. It is estimated that $40 \%$ of the 800,000 orphans in Botswana are children whose parents died of AIDS. Another 30,000 children are vulnerable because of the sickness of one or both parents, who are therefore, unable to care for them.

The epidemic has also heavily affected the youth. The majority of HIV infections occur amongst young people, particularly those between the ages of 13 and 24 [39]. At the end of 2005, an estimated 91,000 children in Botswana had HIV and AIDS and over a half a million children had been orphaned by HIV and AIDS [38]. Botswana is basically divided into three regions and the HIV and AIDS prevalence in these regions is as follows: southern Region, 14. 3\%; Central Region 25\% and Northern Region, $14 \%$.

In summary, since the time that the HIV was first diagnosed in Botswana over 30 years ago, more people continue to be infected on a daily basis [16]. HIV and AIDS surveillance data trends from the National Aids Coordinating Agency show that HIV prevalence has been rising over the years (19901999) and started declining from 2000 to 2005 [34]. The prevalence in the population aged 15-49 rose from $13.8 \%$ in 1996 to $15 \%$ in $1997,16.2 \%$ in 1998 , $16.4 \%$ in 1999 (DHS, 2000) [16]. The prevalence was estimated at $14.4 \%$ in 2002 and currently HIV prevalence is estimated at $14.02 \%$ [16]. The 2004/2005 Demographic and Health Survey found an HIV prevalence of $12.7 \%$ among the 15-49 years old age group in Botswana. This scenario clearly indicates that there is a significant proportion of the population that is with HIV and AIDS.

\section{National HIV and AIDS Policy}

In 2000, Botswana launched its first National HIV and AIDS Policy. The goal of this policy is to prevent HIV infections, to reduce vulnerability to HIV and AIDS, to improve the provision of treatment, care and support for people with HIV and AIDS and to mitigate the socio-economic impact of HIV and 
AIDS on individuals, families, communities and the nation [16].

The objectives of the policy are to: prevent HIV infections; improve delivery of prevention, treatment, care and support services; mitigate the impact of HIV and AIDS on individuals, the family and communities; reduce individual and societal vulnerability to HIV and AIDS through the creation of enabling the environment; strengthening the multidisciplinary institutional framework for co-ordination and implementation of HIV and AIDS programs in the country [16].

Groups suffering from HIV and AIDS in Botswana are discriminated against and this make them vulnerable in the context of HIV and AIDS. These groups include young women and young girls, orphans, widows and widowers, children and young people, the poor, sex workers, prisoners, mobile populations, persons engaged in same sex relations and people with disabilities [16].

The Policy also spells out that to tackle the HIV and AIDS pandemic, people must have the ability to adopt risk-reducing behavior and also be able to utilize existing opportunities to cope with HIV infection and AIDS. Targeted information delivered within a culturally sensitive context can help to increase awareness and knowledge to overcome the stigma, discrimination, myths, beliefs and prejudices associated with HIV and AIDS and sexuality. Mass media supported by interpersonal communication are vital channels to reach out to the largest number of people with accurate, targeted and relevant messages. However adopting and sustaining new behavior requires not only access to information and knowledge but also motivation and support to change attitudes, build skills to practice new behavior and enabling environment in which this new behavior can take place [16].

\section{The Role of Language}

Botswana, just like many other countries in subsaharan Africa, is experiencing a serious HIV and AIDS pandemic, threatening its very survival. Today, despite many years of national response, the impact of HIV and AIDS remains devastating. Illness and death have increased exponentially. HIV and AIDS have left over half a million children orphaned. Community Based Organizations (CBOs), NonGovernmental Organizations (NGOs), Faith Based Organizations (FBOs), public and private sector institutions have all become engaged in various ways in the fight against HIV and AIDS and in activities and interventions that seek to mitigate its impact on individuals, families, communities and institutions.
HIV and AIDS messages are all over, through radios, newspapers, posters, drama, jingles, plays, television, hospitals and many other avenues. Among the many reasons why many people are dying due to HIV and AIDS, is the language of communication for dissemination HIV and AIDS messages. Many of these languages are mostly in English and some are in Setswana yet there are more than 20 languages spoken in Botswana. Most organizations have a common belief that every Motswana understands Setswana. In so doing the masses are denied a chance of getting HIV and AIDS messages through their languages and as a result people are perishing because of lack of knowledge [33]. So it is important that minority languages play their rightful role, HIV and AIDS messages should be culturally sensitive and communicated to the target populations in mother tongue.

Government has shown commitment through the formulation of the HIV and AIDS policy that recognize the role of information education and communication. The policy emphasizes that everyone should have equal access to culturally and age appropriate formal and non-formal HIV and AIDS information communication and education programmes which are adequate and sound; this includes free and accurate information regarding mother to child transmission, breastfeeding, treatment, nutrition, change of lifestyle, and safe sex as well as the importance of respect and nondiscrimination of persons with HIV and AIDS [16]. The policy is written in English and the masses are ignorant of this policy. If the masses are ignorant of the HIV and AIDS policy and messages there is a problem.

\section{Challenges and Opportunities Language and Literacy}

There are still large populations of Batswana that speak and understand only their mother tongue. This means that for these people if anything worthwhile must reach them, it must be written or said in their language. Putting the information in the national and official languages marginalizes minority groups. However, the greatest handicap in this endeavor is that in most Batswana communities where people are only able to use their mother-tongue, the levels of literacy in those languages are quite low. Illiteracy rate in Botswana is at $42 \%$. With all the technological advancements that are taking place in the world, it is quite a challenge to realize that there are many people in Botswana that are illiterate. This is a great setback when it comes to issues of combating HIV and AIDS through information education and communication. 
However, there is a deliberate move by government, faith and non-governmental organizations to empower the masses so that the HIV and AIDS messages get across to them. There are some adult literacy classes which are mounted by government as well as non-governmental organizations. These are yielding good results for those who have gone through such classes are able to read and write. The problem is that these adult literacy classes are conducted in Setswana and not the mother tongue of the target populations. The government of Botswana is committed to literacy programs as spelt out in the HIV and AIDS policy that every person has access to accurate information regarding HIV and AIDS treatment options and shall promote widespread literacy campaigns and access to information on where and how to access treatment, care and support.

\section{Language of Communication in Development of Materials}

Language can be a barrier to HIV and AIDS dissemination of messages if not properly handled. Using a foreign language in delivering information on HIV and AIDS in a language which the majority of the population is not literate in is a challenge. Higher levels of understanding can only be attained if an appropriate language is used to communicate information and impart knowledge on HIV and AIDS. Language is most appropriate when a majority of the people is able to share information and knowledge freely using that language. So far the most commonly used languages in the writing and dissemination of HIV and AIDS messages are English and Setswana [11].

When it comes to communication issues of HIV and AIDS, it is inevitable that literary means of communication be used. It is lamentable, however, to notice that with regard to the proliferation of HIV and AIDS literature in the country, there are fewer materials available in other local languages apart from English and Setswana. In so doing the majority of the people are prevented from getting HIV and AIDS messages.

Most materials on HIV and AIDS have been developed in English. Later they are translated into Setswana. But when you check the people involved in the development of the materials you find they are Batswana. The biggest question is, if the institutions they represent realize the importance of communicating through the people's languages and that the target groups or beneficiaries of that particular information are not literate in English, why develop the materials in English and waste time translating? Because through that process meaning gets lost. Why not develop the materials straight into the languages of the target populations.

However, the arguments have been, Setswana and other local languages do not have adequate vocabulary, it is difficult to express concepts of some highly specialized domains like, health, management, justice etc. terminologies can be developed to make up for this though it is not an easy task.

\section{Religious Institutions and the Foreign Language Literacy Phenomenon}

In today's world with the HIV and AIDS pandemic most people attend general prayers, fellowships and healing sessions organized by different faith groups with the hope to be healed both spiritually and physically. Apart from teaching the word of God, religions teach people to change behaviors, to transform for self-advancement [8]. Religions leaders preach about being responsible citizens, participation in various developmental activities, such as politics, health education; they also preach against domestic violence through word of "God". An influx of people from all walks of life attends these functions. Religious gatherings have become the most appropriate forums, in my view, for delivering the HIV and AIDS messages of transformation to the people.

One of the most messages delivered in various faith groups is to avoid committing adultery and this is the same message that is all over, asking people to remain faithful; to stick to one partner. Religious and various social institutions are fighting for the same cause and preventing HIV and AIDS is one of their biggest challenges. HIV and AIDS is affecting and killing the most productive group in the development of the nations.

However, most preachers of Pentecostal Faith Missions in the country prefer to use English, a language that is foreign to the congregation, instead of using a language most appropriate to the audience. As a result most people do not understand the messages or they misinterpret them. Of course some use interpreters, but at the speed preachers go, coupled with lack of skill, expertise and training on the part of the interpreters, one questions and the authenticity of the message in the target language. Interpreters whom they use distort the messages since they are not professional interpreters and meanings in translation are not in one to one correspondence with the source languages. Consciously or unconsciously these preachers ignore their purpose, to get the 
message across to the audience, be it religious or on HIV and AIDS.

\section{Language in the Media}

As mentioned earlier, in Botswana, there are a total of 87,000 deaths every year and every minute someone dies of HIV and AIDS [16]. The situation is worsening every year. In response to this rampant situation there has been a huge turn to the media. The media acts as a way through which HIV and AIDS information education and communication is disseminated. The media plays the role of promoting change of behavior, condom use, abstinence, dangers of the disease; how it is contracted, voluntary testing and counseling and reduction of HIV and AIDS related stigma among others. The media disseminates HIV and AIDS messages through various channels such as the radio, television, video, print media, internet etc. However, there are language related challenges that impinge on successful use of the media.

Evidence of language related media challenges to HIV and AIDS message dissemination is noted in the results of a survey carried out by in Khakhea in Kgalagadi [11]. It is reported that one of the modes access to HIV and AIDS information by members of the communities in Kgalagadi area is the radio. The statistics show that the radio $(41 \%)$ is the most available and the easiest mode of access to information on HIV and AIDS in the area. However, this is not effective because the language commonly used on the radio is Setswana and not Shekhalahari a language spoken by $98 \%$ of the people in that area and in their homes.

Another survey that shows evidence of media language related challenge to media dissemination of messages was conducted by Manda. Manda observes minimal use of local languages on the public broadcaster, Botswana television (Btv), the only TV station in the country. Botswana television only uses English and Setswana while other languages are just voiced over in the interviews. Batibo also observes that Radio Botswana 1 (RB1) broadcasts in languages other than English and Setswana for $6.2 \%$ of the weekly broadcasting time [11]. Out of the $6.2 \%$ Ikalanga gets $1.2 \%$, Shekhalahari gets $1.1 \%$, Sesarwa, Setswapong, Sebirwa and Setebele get $1 \%$ each. However, very little or none of the messages are on HIV and AIDS [11].

In the print media, Mmegi newspaper and the daily news, all the pages are $100 \%$ in English. Setswana, the national language is never used $(0 \%)$ nor are the other Botswna languages (0\%) [11]. Furthermore, the week-end papers like weekendPost.

\section{Direct Language in HIV and AIDS Message Dissemination}

The use of direct language scares or puts off target audience. This is a challenge to effective dissemination of HIV and AIDS messages are exemplified in the research findings of Batibo and Mosaka [11]. In khakhea area, Kgalagadi, many people do not like to listen to the messages on the radio because most times it uses direct language and the HIV and AIDS are aired when children are around or before they go to bed. As a result the adults feel embarrassed because culturally, sex issues are not discussed in public and more so in the presence of children. This has resulted in people's negative attitudes towards the HIV and AIDS messages aired on the radio. Most people do not listen to the HIV and AIDS related programs which to them are obscene and therefore embarrassing, hence failure to access information on HIV and AIDS. Most of the people would opt for use of euphemistic or indirect language. Most people in the area access information from friends (14\%) who are brave to listen to the messages. Much as this might help but there is a danger of giving each other wrong information because these friends are not experts in the area of HIV and AIDS. However, with quite a lot of civic education, the situation might change for the people will see the need for straight talk to access information on HIV and AIDS and therefore listen to the radio in the presence of the children on issues of HIV and AIDS if they are to survive the pandemic.

\section{Language of the Youth in HIV and AIDS Information Education and Communication}

The groups that are most affected with HIV and AIDS are the youth. The language, particularly the terms and expressions used in communicating to the youth have to be fully understood by the target groups. If this is not the case, the intended outcome will not be realized. The question is, "Do we use youth language or ordinary day to day language?"

Children and young people are expected to have access to youth friendly sexual and reproductive health information and education including HIV and AIDS information appropriate to their age, to equip them with knowledge and skills to protect themselves from HIV and AIDS and other sexually transmitted infections (STIs). It has also suggested that since that since the majority of HIV infections in Botswana occur amongst young people, greater emphasis should be placed on HIV and AIDS education in schools. 
However, HIV and AIDS is still a taboo subject in many communities within Botswana and discrimination is common. Many have difficulty discussing the subject with their families and some support groups do not meet openly. As a result, few people with HIV and AIDS make their status known. However, government has put in place mechanism to ensure that HIV and AIDS and STIs prevention, treatment, care and support and impact mitigation services are accessed by all without discrimination, including the youth.

Results of research carried out by chisoni, in primary schools among primary school pupils aged 7 13 years in Francistown and Gaborone city show the challenge to straight talk and discussing sexual matters with pupils in school [13]. Chisoni found out that $65-85 \%$ of the youth misunderstood the meanings of euphemistic expressions used by teachers in HIV and AIDS education. These were like go robalana (sexual intercourse vs. sleeping over one another), sekausu (sexual intercourse vs. socks), go nna mmogo (sexual intercourse vs. staying together). And $85 \%$ of primary school skills teachers interviewed in Francistown indicated that discussing sexual issues openly with pupils becomes embarrassing hence they opt for indirect, euphemistic expressions. The government of Botswana is also of the opinion that it is becoming difficult for life skills teachers to discuss sex topics openly with their pupils (Ministry of Education, 2008).

This is because the use of explicit reference to human genitalia (for example; polo (penis), phuna (vagina) and/or sexual behavior, 'go robalana' (sexual intercourse) is culturally inappropriate in the Botswana context. Reference to such matters is done indirectly or using euphemisms or youth language with restriction to one age group and sex. It is not Botswana culture to use direct words for sexual matters [32]. Use of euphemism is characteristic of many cultures in African communities since sex is deemed a taboo subject (Ogechi, 2005). Issues of sex are face threatening among most Batswana.

However, straight talk is encouraged for the sake of clarity. With the current HIV and AIDS epidemic, it has become necessary to engage in what is known as 'straight talk' about sex organs/or sexual behavior even with school children. This is because the use of euphemism does sometimes fail to convey the message clearly (kamwendo, Bwanali, Nyirenda and Kachiwanda 2001). The use of direct and noneuphemistic language during dissemination of HIV and AIDS messages ensures that we impart the right and accurate information to the intended audience. Mkandawire observes that use of euphemistic expressions in communicating HIV and AIDS messages to the youth because everybody is afraid of using obscene language has led to many youths trying things they do not understand [30]. The language that could effectively communicate HIV and AIDS messages has been ignored consciously or unconsciously. If use of euphemistic expressions continues we should not expect the youth to grasp the messages fully for they will have half-truths.

The use of simple and straightforward noneuphemistic expressions could be fundamental in checking the prevalence of HIV and AIDS amongst the youth in Botswana. This is because the youth in various age groups, have different levels of understanding due to experiences in life and levels of education. One factor that has been ignored by some advocacy in Botswana in programs targeting the youth is the language used in relaying these messages. It seems the use of euphemistic expressions therefore is one of the reasons contributing to the spread of HIV and AIDS amongst the youth. Using euphemisms is not effective unless the intention is to hide information from the youth. Therefore, HIV and AIDS advocacy campaigns have to develop or use language considered as explicit and obscene, but as far as it is carrying the exact meaning of what is expected by the encoder and at the same time comprehended and/or interpreted as intended by the recipient, it is the appropriate language to use.

There are interesting research findings that contradict the use of direct language. Thebeetsile, a student at the University of Botswana, reports that at Tonota College of Education (TCE), due to the use of direct language in peer education some youth feel intimidated by the language in peer education, thus messages on HIV and AIDS are inappropriate [37]. Peer education at Tonota College of Education employs the concept of straight talk that is use of direct language. Biological terms for instance penis ('polo' in Setswana), vagina ('phuna' in Setswana), and sexual intercourse (go robalana in Setswana) amongst others are used. The straight talk concept is a good ideology, however, it undermines the fact that the youth are not a homogenous group therefore are not all comfortable with the use of direct language. Their reaction to such messages is twofold. Some students feel the use of the direct language in HIV and AIDS peer education campaigns act as forums where 'foul or obscene language is condoned and promoted. Some students therefore shun away from attending HIV and AIDS peer education campaigns. For "Born again students" the use of direct language is contradictory to their religious values and morals. The shunning away of these students is a backdrop in the fight against HIV and AIDS pandemic because the fight requires involvement of all the youth considering the fact that the youth are the much affected age group. Some students look upon these 
peer education gatherings as a form of entertainment for them to hear 'foul or obscene language.' Therefore among fellow peers it is an amusement phenomenon, a moment for poking fun. Whenever direct language is used there is lack of seriousness, students are always whistling and shouting, more especially the male students [37]. Thus, although the HIV and AIDS has been a major issue of public debate within the University of Botswana, in particular college of Education, it is still rather difficult to talk about HIV and AIDS messages without evoking different attitudes, emotions and feelings due to the employment of the concept of straight talk.

\section{Cultural and Traditional Values}

The campaigns in Setswana language are also hampered by cultural and traditional values. Culturally in Botswana it is a taboo to talk about sex in public, let alone mention the human anatomy. The government through the Ministry of Health and NGOs like Population Services International in Botswana (PSI Botswana) are campaigning and promoting for more open dialogue about sexuality. PSI Botswana which sponsors 'Youth Alert' programmes on radio as well as 'mareledi' program on television puts emphasis on 'straight talk'. Contrary to their emphasis and campaign on 'straight talk' what is being used on these programs is not 'straight talk'. Culturally describing sexual act is cursing or undressing someone.

The question that everybody would have is why PSI Botswana is using euphemistic terms when together with government, are advocating for 'straight talk' or 'being in the open?". The earlier booklets by PSI Botswana had real words for private parts like 'Polo' for 'penis' not 'thobolo' (gun or weapon for man, etc) as the case is now. Someone must have censored them into culturally insensitive. This may also be a result of societal pressure to observe human decency. The word 'penis' in English, for example, does not sound offensive or a taboo but the Setswana version is. It is so much of a taboo that it is not even mentioned in private. The mere mention of it seems as if one is insulting someone. Therefore PSI has resorted to using euphemisms because they are becoming sensitive to cultural values. This is a challenge, unless we change our culture, which we cannot do, the use of euphemisms or indirect expressions will include information and not help in mitigating HIV and AIDS.

\section{Language and Gender Stereotyping}

Language is the medium that tells us what our society thinks about males and females and this is very much clear in some HIV and AIDS messages. Females and males are stereotyped in HIV and AIDS messages on radio and media print houses. This affects the female and male genders. We need to find out what can be done to turn round this language use which stereotypes the female and male in HIV and AIDS messages.

Gender stereotyping is drawn mainly by looking at the semantic components of the words which have been used in the messages. Women are stereotyped as being weak, emotional, dangerous, dependent and submissive; they may get hurt and do nothing about it. On the other hand, males are stereotyped as being strong, rational, domineering and independent among others. Some may take it as true and act on females as their prey, rape and batter them. At household level this gender discrimination and stereotype may lead to couples exposing themselves to a risk of contracting HIV and AIDS whilst trying to run away from the gender based violence. Some males may turn to having extra-marital affairs with other women and engage themselves into unsafe sex. The females may also do the same.

Therefore what started as an innocent HIV and AIDS message to inform people on what HIV and AIDS is all about reaches the people with some assumptions and interpretations and may place them in a situation whereby they are prone to contracting HIV and AIDS.

To deal with the problem of stereotyping in HIV and AIDS messages; the following is proposed: Language should be used to come up with messages that are gender sensitive and with gender stereotypes. There should be an effort through language to change people's mindset against gender stereotyping, with time then the problem of gender stereotyping in HIV and AIDS messages will be eradicated.

\section{Language and Target Groups}

Face to face interactions and any literature on HIV and AIDS should be in the main language of the area if the target populations are too readily understand and positively accept and respond to the HIV and AIDS messages. Words and expressions take on different shades of meaning in different languages within the same country. For example, a swear or obscene word in one language could be a perfectly good and non-embarrassing word in another. For example, 'go itaya' in Setswana means to 'hit' whereas in Sekgatla means 'sexual act'. 'Go 
jana' (sexual act) in Setswana is a taboo word whereas in Sekgatla is not. In certain parts of Botswana, for example, Kgatleng and Tswapong some swear words including names of private parts are used to express happiness especially among the older generation. If HIV and AIDS education campaigners are not aware of all this, the beneficiaries of the HIV and AIDS education campaigns may end up shunning away from such meetings. Therefore realizing the different connotations that messages could have depending on the language being used and the target population, it would be appropriate to use the minority language of a group to which the HIV and AIDS message is being communicated. In so doing people receive with a positive attitude and act on it in a similar manner. Information education and communication in a foreign or second language creates a gap and lead to misconception.

\section{The Role of Interpreters in the Use of Unfamiliar Language}

Language policy in Botswana recognizes English as an official language and Setswana as a national language out of the 28 indigenous languages of Botswana. Because of the status given to Setswana, it has been assumed as a language of the masses and that messages communicated through it will be readily understood and responded to by everybody in the country. Results of a research conducted by Batibo and Mosaka to find out language use in various domains like the home, market places, hospitals etc show that this assumption is not a correct one and therefore a challenge to effective communication of HIV and AIDS messages [11]. Among 200 households in six villages the survey revealed that Kgalagadi is one of the areas where most of the population cannot speak and understand Setswana because $98 \%$ of the people speak Shikhalahari most of the times. And $17 \%$ use Setswana at school, $47 \%$ could not tell which language is used at school because they have never been to school themselves. Setswana which is used for dissemination of HIV and AIDS messages is acquired at school. Most people do not have a chance to acquire Setswana language which is most often used for HIV and AIDS campaigns.

Thus people in this area have problems with HIV and AIDS messages that are transmitted through Setswana, the language they do not use. This means that these people are misinformed on HIV and AIDS and many other issues. Similar observations were made in research findings Francistown and Masunga where it is assumed the communities speak and understand Ikalanga [11]. Kayabazinthu, in another study in Nata, a Sesarwa speaking area, where people could not understand Setswana, made a similar observation. In these areas, facilitators who cannot speak Sesarwa use interpreters. Use of interpreters is a threat for it might distort the information. Those who interpret are not trained interpreters and they lack interpretation skills. In addition, clients feel uncomfortable with the presence of a third party to seek or reveal information on HIV and AIDS as well as STDs. To ensure that everyone has access to information, therefore, familiar (minority) languages must be used.

Mtenje argues that "we cannot 'communicate' to the people or indeed understand them unless we go through a language which is closest to their hearts namely their native or most familiar language." [33] This means that the use of Setswana to disseminate HIV and AIDS messages in Botswana has not targeted the minority communities. We cannot make communities that speak minority languages fight something they do not understand. For them to fight better they should be equipped with the relevant information on HIV and AIDS through a language they speak and understand, in this case native language.

\section{Empowerment of Minority Languages through Standardization and Terminology Development}

The fact that most minority languages are not yet standardized is a challenge. Botswana languages lack standardized orthography and terminologies that can be used in schools, official communication and in publishing. HIV and AIDS have brought with them several strange words that never existed in the local languages, some of them scientific and health related. Some attempts have been made to translate or borrow but this has left the intended meaning unclear. For example, in Setswana, 'HIV and AIDS' is AITS. Anti-retroviral (ARV), dipilisi and Condom is 'sekausu', (socks).

Out of the twenty eight local languages only three languages (Ikalanga, Seyeyi, Shekhalahari) have a standardized orthography and therefore it is a hindrance in the use of other minority languages. Lack of standardized orthographies leave broadcasters and other development communicators as sole experts in coming up with the orthography of the language. 


\section{Conclusion}

HIV and AIDS is spread by certain types of human behaviors, therefore it can be controlled by changes in those behaviors. What is needed is continued information education and communication to all sectors of society to promote interventions that can reduce high-risk sexual behaviors, treatment and control of other sexually transmitted diseases, ensure that human rights are respected, care and mitigate the problems of those already infected with or otherwise affected by the epidemic. Use of minority languages may be used in the media like radio drama and information dissemination activities. When HIV and AIDS messages are carried out in the local languages of the potential beneficiaries, they are communicated more effectively to such users. If Setswana or English is used where it is not spoken and understood the public media has no effect because the people do not access the information. The paper therefore argues that to make sure that everyone has access to HIV and AIDS information, minority languages spoken in different areas of Botswana must be used when holding HIV and AIDS education campaigns and providing services to the people infected by HIV and AIDS because these are languages that carry people's culture and are best understood by the communities.

\section{References}

[1] Abrams, M. H (1961). A Glossary of Literary Terms. Cornel: Cornel University.

[2] Anderson et al (1997). Language in Botswana. Gaborone: Longman.

[3] Anshen, R. N. ed. (1971). Language: An Inquiry into Its Meaning and Function. Kennikat Press, New York.

[4] Antilla R. (1972). An introduction to Historical and Comparative Linguistics; New York, Macmillan Publishing Co. Inc.

[5] Asher, R. E (ed in chief) (1994). The encyclopedia of Language and Linguistics: Vol 9. Edinburgh: Pergamum Press Ltd.

[6] Ayto, J. (1993). Euphemism, over 3000 ways to avoid being rude or giving offence. London: Bloomberg Publishing Ltd.

[7] Archibugi, D. (2005) The Language of Democracy: Vernecular or Esperanto? A Comparison between the Multiculturslist and Cosmopolitan Perspectives. Political Studies 53 (3), 537-555.

[8] Bahai International Newsletter. (2004) 'One country: perspective Literacy and Development,' 1, (16), 1 April-
June (http://www.ufbahai.org/articles/literacy-and development), last accessed $16^{\text {th }}$

[9] Batibo H. M. and Kopi M.M (2004a). A Socio Linguistic study of Euphemistic and Idiomatic Expressions used in HIV/AIDS Speech in Setswana.

[10] Batibo H. M, and Kopi M. M (2004b). A Study of the Sex related Euphemisms in Setswana.

[11] Batibo, H. M. (1997). The fate of the minority languages of Botswana. In B. Smieja and M. Tasch (eds) Human contact through Language and Linguistics (pp. 24352). Frankfurt: Peter Lang.May, 2008.

[12] Bertha, E. (2008) Career of the Week. The Weekend Nation. Januuary. The Youth and Language Use in HIV and AIDS messages: A case of Peer Education on HIV and AIDS

[13] Chisoni, F. (2008). The Use of Local NonEuphemistic Expressions. A paper presented at University of Malawi, centre for Language Studies Research Dissemination conference on Language and HIV and AIDS - Hippo View Lodge 28-31 Jnuary 2008.

[14] Durkheim, E (1963). Incest: The Nature and Origin of the Taboo. New York: Lyle Stuart.

[15] Ginn, D. S. (1987) The HIV/AIDS information crisis: confluence of the roles of information creator, seeker and provider. In Bulletin of the Medical Library Association, Oct 4 (75).

[16] HIV/AIDS in Botswana. (2003). Estimates of the prevalence of infection and implications. National Aids Commission Agency (NACA).

[17] Holder R. W (1990). A Dictionary of Euphemisms. Oxford; University Press.

[18] Hornby, A. S (1994) Advanced Learners Dictionary. London: Blackwell.

[19] http://www.peevish.co.uk/slang/2003, Euphemistic Expressions from Encyclopedia

[20] http://www.mmodychurchofchrist.com/Articles/article Euphemisms Html page 1- 3 by Tom Roberts

[21] http://www.uta.fi/fast/usi/ref/ Euphemism. Html page 2 of 2 from American English Reference file.

[22] http://www.en.wikipedia.org/wiki/ Euphemisms page 1- 3 from encyclopedia by Stephen Pinker

[23] http://www.eu.wikipedia.org/wiki/ Talk page 1-7 from encyclopedia by Sonia Robbins

[24] http://www.hiceducation.org/ Educ_Proceeding/ katherine $\% 20 \mathrm{~m} \%$ Houghton. Pdf: ma literature review Euphemism Research. By Katherine Haughton 
[25] Hudson (1980). Sex and sexuality: Questions and answers for counselors and therapists. London; Philadelphia: Whurr

[26] Kamanga, M. and Shame-Kalilombe, E. (2008). Minority Languages: A tool for HIV and AIDS fight. A paper presented at University of Malawi, centre for Language.

[27] Kishindo, P. J. (2006) 'Language and Health Services Provision.' In: The Lamp, No. 62, November-December 2006, 20-21.

[28] Kishindo, P. J. (1994). The impact of a national Languge on Minority Languages: The Case of Botswana. In: Journal of Central African Studies, 2, (12), 127-150

[29] Mogapi, K. (1985). Ngwao ya Setswana. Gaborone: Gaborone Printing Works Pty.

[30] Mkandawire, K. (2007). HIV/AIDS and the Media. Daily Times (http://programmecommunication.blogsport.com/), last accessed, June 2014.

[31] Ministry of Health and Population, Botswana. (2004) 'Treatment of AIDS, the two year plan to scale up antiretroviral therapy in Botswana.

[32] Moto, F. (2004). Towards a study of the Lexicon of Sex and HIV/AIDS, Nordic Journal of African Studies, 3, (13), 343-362

[33] Mtenje, A.D (2002). The role of language in national development. The case for local Languages. Inaugural Lecture, Chancellor College, Zomba.

[34] National Aids Coordinating Agency (2003). Botswana national HIV/AIDS Policy.

[35] Ogoche, N. O. (2005). The Languge OF Sex and HIV/AIDS among university students in Kenya. Stichproblem. Wiener Zeitschrift fur kristische Afrikastudien No.9,5:123-149.

[36] Roberts, Amanda. (2000). AIDS: your questions answered. Ibadan: Heinemann

[37] Thebeetsile, J. (2006). A Sociolinguistic study of euphemistic expressions used in Setswana. (MA Thesis), University of Botswana, Faculty of Humanities, Department of African Languages and Literature, Gaborone

[38] UNAIDS. (2006). Report on global AIDS epidemic, Annex 2: HIV/AIDS estimates and data, 2005.

[39] UNAIDS/WHO. (2006). Report on the gobal AIDS epidemic.
[40] UNICEF, UNAIDS, WHO (2002). Young People and HIV/AIDS: Opportunity in Crisis

[41] UNICEF, et al. (2002) young People and HIV and AIDS Opportunity in Crisis. UNESCO (2003a). UNESCO Red Book of Endangered Languages: Africa (data supplied by $B$. Heine and M. Brenzinger). Tokyo: International Clearing House for Endangered Languages (ICHEL). http://www.tooyoo.l.u-tokyo.ac.jp/redbook/index.html 